\title{
BIOGÉOGRAPHIE DU SILURE GLANE (SILURUS GLANIS) : CAUSES HYDROGRAPHIQUES, CLIMATIQUES ET ANTHROPIQUES.
}

\author{
O. SCHLUMBERGER, M. SAGLIOCCO ET J.P. PROTEAU \\ CEMAGREF, U.R. Ressources Ichtyologiques en Plans d'Eau, \\ BP 5095, 34033 Montpellier cedex, France.
}

Reçu le 26 juin 2000

Accepté le 18 octobre 2000

Received 26 June, 2000

Accepted 18 October, 2000

\section{RÉSUMÉ}

La répartition actuelle du silure glane (Silurus glanis) en France et en Europe résulte de la combinaison de facteurs à la fois hydrographiques, climatiques et anthropiques. Des données paléontologiques montrent que l'espèce faisait partie de l'ichtyofaune française (bassin du Rhône) avant d'être éliminée par les glaciations. Le réchauffement climatique qui a suivi (10 000 av. JC) et l'existence d'interconnexions entre, d'une part, des tributaires de la mer Noire et de la mer Caspienne et d'autre part ceux de la mer Baltique et de la mer du Nord lui ont permis de rapidement coloniser le Nord de l'Europe occidentale. Deux périodes de transplantations-introductions de l'espèce, à but économique, sont identifiées. Une première vague d'introductions hors du bassin danubien semble avoir eu lieu dès le Moyen-Age (lacs de Suisse), suivie par une deuxième période débutant vers 1800, et se poursuivant actuellement. Les effets combinés de ces introductions et des dégradations du milieu d'origine anthropique ont induit un décalage de l'aire de distribution vers le Sud et le Sud-Est de l'Europe (Italie, Espagne), tandis que ne subsistent en Suède, Carélie, Russie et Estonie que des populations relictuelles.

Les résultats présentés ici, pourraient aussi concerner d'autres espèces de poissons (Percidés, Cyprinidés), qui, après avoir bénéficié pendant les glaciations des refuges constitués par les bassins de la mer Noire et de la mer Caspienne, ont pu (re-) colonisé l'Europe continentale, de manière « naturelle » et avec l'aide de l'Homme.

Mots-clés : biogéographie, Europe, Silurus glanis, fossiles, populations relictuelles.

\section{BIOGEOGRAPHY OF THE SHEATFISH (SILURUS GLANIS) : HYDROGRAPHICAL, CLIMATIC AND ANTHROPIC CAUSES.}

\section{SUMMARY}

The biogeography of the sheatfish (Silurus glanis) results from the combined impacts of hydrogeography, climate and man. Paleontological evidences prove that the species was present in France (Rhône river) before the glaciations. The improvement of the climatic conditions (from $10000 \mathrm{JC}$ ) and hydrographical interconnexions between tributaries of the Black and Caspian Sea basins, and between the Baltic and North sea made possible the colonization of the Northern part of Western Europe by Silurus. Two phases of transplantation-introduction from the Danube area are known, both linked to the economical interest of the species. The combined effects of these introductions and the 
anthropogenic degradations of its environment induced a shift in the distribution area of the fish : a strong rarefaction in Northern Europe (Sweden,...), and introductions in Italy, Spain and South-West of France. The results presented in this paper can concern other fish species than S. glanis (Percids, Cyprinids) which may have followed the same paths from their initial refuges around the Black and Caspian seas to (re-) colonize the continental Western Europe after the glaciations.

Key-words : biogeography, Europe, Silurus glanis, fossils, relictual populations.

\section{INTRODUCTION}

La distribution au cours du temps du silure glane en Europe de l'Ouest telle qu'elle nous est connue par des restes fossiles ou des débris osseux datant de la préhistoire ou du haut Moyen-Age, par l'existence d'une population relictuelle isolée par un bras de mer (Sud de la Suède), par la disparition de ce poisson dans le Rhin puis sa réapparition un siècle plus tard, demande que l'on fasse la part de ce qui est dû respectivement aux effets climatiques, à la paléohydrographie et aux causes d'origine anthropique.

\section{DISTRIBUTION DE L’ESPÈCE}

La distribution naturelle du silure glane est liée aux exigences biologiques de l'espèce vis à vis de l'hydrologie et de la température de son milieu de vie. Le silure vit dans les eaux calmes, profondes et turbides : zones à barbeau et brèmes des cours d'eau de plaine, et dans les grands lacs, où il recherche les abris sous berge ou sur le fond (racines, éboulis, souches). Son régime alimentaire peut être qualifié de carnivore opportuniste (PINTER, in VALLOD, 1987).

L'espèce est considérée à la fois comme rustique, puisqu'elle supporte une large gamme de températures $\left(3\right.$ à $\left.30^{\circ} \mathrm{C}\right)$, des teneurs faibles en oxygène dissous (FORGUE et al., 1989 ; MASSABUAU et FORGUE, 1995), et thermophile : la température optimale pour sa croissance est de $25-27^{\circ} \mathrm{C}$ (HILGE, 1985). A partir de $15^{\circ} \mathrm{C}$, la prise de nourriture est active et la croissance devient notable. Pour que sa reproduction ait lieu chaque année, il est nécessaire que la température de l'eau dépasse $20^{\circ} \mathrm{C}$ pendant 2 à 3 mois si l'on se base sur les exigences thermiques de la femelle pour sa maturation en pisciculture d'étang (PROTEAU et al., 1994). La ponte, dans un «nid» de racines, est gardée par le mâle.

\section{Distribution jusqu'à la fin du XIX ${ }^{\text {ème }}$ siècle}

A la fin du $19^{\text {ème }}$ siècle en Suède, une petite population de silure glane subsistait dans quelques fleuves côtiers au Sud de Stockholm (NATHANSON, 1986, 1987). GILTAY (1931) indique la présence de l'espèce dans le Sud-Est de la Finlande. En réalité, les dernières captures en lacs faites en Finlande datent de 1864-66 (KOTIRANTA et al., 1998). Il a disparu du lac Sorö (Danemark) depuis le début du 19ème siècle, mais aux Pays-bas était encore présent dans la région d'Amsterdam («HAARLEMMERSEE »; GILTAY, 1931 ; VOLZ, 1994). En Russie et Europe centrale, le silure était signalé dans les lacs Onega et Ladoga ainsi que dans la Neva (Saint Pertersbourg) qui relie le lac Ladoga au Golfe de Finlande. II était présent également dans les bassins hydrographiques de la mer Noire (Danube, Dniepr, Don), de la mer Caspienne (Volga), de la mer d'Aral (CUVIER et VALENCIENNES, 1839 ; GILTAY, 1931). Ces mêmes auteurs mentionnent sa présence dans des fleuves tributaires de la mer Baltique (la Spree, la Memel, la Vistule, la Pregel) et de la mer du Nord (dans l'Elbe). 
II était «pêché dans le Rhin », indique VALLOT (1837), mais encore inconnu en France pour CUVIER et VALENCIENNES (1839), où il sera introduit un peu plus tard, en 1857 (KEITH, 1998).

Plus ancien, un ouvrage de BALDNER consacré à la faune d'Alsace publié en 1666 présentait l'image d'un silure glane vu sur le marché aux poissons de Strasbourg. CUVIER et VALENCIENNES (1839) précisent que l'animal avait été capturé dans l'Ill, qui rejoint le Rhin à Strasbourg. Toujours CUVIER et VALENCIENNES (repris ultérieurement par d'autres) mentionnent la présence du silure dans la Moselle, à l'époque romaine, en se basant sur un poème d'AUSONE (4ème siècle après JC) dédié à ce fleuve. Mais pour certains, le terme silurus utilisé n'est qu'une forme poétique pour désigner un poisson pisces d'espèce quelconque mais de grande taille (en l'occurrence, silure glane ou esturgeon $A$. sturio).

Des populations existaient en Suisse dans les lacs de Bienne, Morat et Neufchâtel (GILTAY, 1931), et plus anciennement, le silure avait été capturé dans le lac de Lucerne, le lac des Quatre Cantons (une capture unique en 1601) et celui de Zurich, après introduction de l'espèce à la fin du XVII ${ }^{\text {ème }}$ siècle (HARTMANN, 1827 ; CUVIER et VALENCIENNES, 1839 ; FATIO, 1890). Le lac de Constance est le seul lac où l'introduction du silure par l'homme soit à la fois attestée et ait réussi ; le record local est un individu de $75 \mathrm{~kg}$ pris au filet en 1882 (SCHMID, 1996).

MOHR (1957) indique la présence de l'espèce dans le Nord-Est de la Grèce (région de Thessalonique) et en Turquie : près d'Iznick et Nicée (Ouest du pays), ainsi que dans le lac de Cildir (à l’Est, près de la frontière géorgienne).

\section{Distribution actuelle}

Dans le nord de l'Europe, la distribution du silure ne s'étend pas au delà de l'isotherme de $16^{\circ} \mathrm{C}$ (juillet) qui englobe le Sud de la Suède, de la Finlande et de la Carelie russe (température moyenne annuelle de ces régions: $6^{\circ} \mathrm{C}$ ). De telles conditions climatiques ne permettent que la survie de petites populations fragiles:

- les femelles peuvent ne pas se reproduire tous les ans (NATHANSON et al., 1987) faute d'accumuler chaque année une somme de degrés $x$ jours suffisante pour assurer l'ovogenèse et la maturation des ovules.

- la survie hivernale des juvéniles est un facteur qui limite fortement le développement de la population. NATHANSON (1989) indique que la taille de $25 \mathrm{~cm}$ (soit un poids inférieur à $100 \mathrm{~g}$ ) n'est atteinte qu'à l'âge de 3 ans en Suède. II est vraisemblable qu'à leur premier hiver, les juvéniles ne dépassent guère une longueur de $10 \mathrm{~cm}$ (environ $10 \mathrm{~g}$ ). Or, même dans le Centre de la France et dans un milieu aussi favorable qu'un étang de pisciculture, la survie hivernale des juvéniles de moins de $40 \mathrm{~g}(15 \mathrm{~cm})$ est inférieure à $40 \%$ (HENNEQUART, comm. pers.).

Actuellement, l'aire de répartition de l'espèce s'étend du Sud de la Suède jusqu'à la plaine du Pô. Tous les bassins hydrographiques français sont désormais colonisés. Vers l'Est, l'espèce est présente jusqu'à l'Oural, dans les bassins hydrographiques des tributaires de la mer Noire, de la mer Caspienne et de la mer d'Aral. Vers l'Ouest, le silure est présent dans le Sud de l'Angleterre, ainsi qu'au delà des Pyrénées (un site en Espagne). Ses exigences thermiques pour sa reproduction $\left(22^{\circ} \mathrm{C}\right.$ pour la ponte, en Suède comme ailleurs en Europe ; HORVATH et al., 1984 ; NATHANSON, 1989 ; SCHLUMBERGER, 1997) limitent son extension vers le Nord mais la facilitent dans les régions méridionales. 


\section{INFLUENCES HYDROLOGIQUES ET CLIMATIQUES (PASSÉES ET RÉCENTES)}

La présence du silure telle qu'elle est attestée dans différentes régions d'Europe avant et après les grandes glaciations de la fin du Tertiaire et début du Quaternaire nécessite que l'on s'intéresse aux interconnexions qui ont existé entre les bassins hydrographiques du Danube, du Rhin et du Rhône. L'Europe orientale (mer Noire, avec le Danube ; mer Caspienne, avec la Volga) parait avoir constitué le « réservoir » ou le foyer à partir duquel le silure a re-colonisé l'Europe occidentale après les glaciations (PERSAT et KEITH, 1997).

\section{Les traces anciennes de silure : fossiles et archéologiques}

Des restes fossiles de silure Silurus $s p$. datant d'avant les grandes glaciations ont été trouvés sur deux sites d'Europe datés de -8 à -5 millions d'années (Miocène supérieur ; ère Tertiaire) :

- dans le bassin du Danube, près de Vienne (Autriche), où ils étaient associés à des Cyprinidés (genres Scardinius, Rutilus, Palaeocarassius, Tinca, Barbus) et des Percoïdés (GAUDANT, 1994),

- dans le bassin hydrographique du Rhône, où l'espèce a été identifiée en Ardèche, accompagnée de Barbus, Leuciscus, Blennius et Perca (MEIN et al., 1983).

Des rayons osseux de nageoires pectorales de Silurus (ou de Parasilurus) trouvés près de Perpignan sont un peu plus récents (Pliocène : - 6 à - $3 \mathrm{Ma}$ ) ; ils sont conservés à l'Université C. BERNARD de Lyon (département des Sciences de la Terre ; BRANA et RIGAUD, 1997).

En revanche, le bassin du Rhin n'a pas livré de traces de la présence du silure aussi anciennes, soit faute d'avoir découvert un site où des restes identifiables se seraient conservés, soit parce que l'espèce ne fait partie de l'ichtyofaune du Rhin que depuis la fin des grandes glaciations. En effet, dans le Nord-Ouest de l'Europe continentale, l'espèce n'apparaît dans les fouilles archéologiques que vers - 10000 ans : des vertèbres de silure ont été trouvées près de Namur, en Belgique (rive droite de la Meuse) et datées du Magdalénien (entre - 14000 et - 10000 ans ; GILTAY, 1931). Ces informations doivent être rapprochées de ce que nous savons des interconnexions entre les bassins hydrographiques du Danube, du Rhin et de l'Aar et celui du Rhône et de leur évolution à la fin du Tertiaire, période où d'importants mouvements de l'écorce terrestre ont eu lieu dans la région (PETIT et al., 1996).

\section{Paléo-hydrographie des bassins supérieurs du Danube, du Rhin et du Rhône}

De nombreux travaux étudient les évolutions successives des bassins versants amont du Rhône, du Rhin, du Danube et les interconnexions qui ont existé à une époque ou une autre. Nous nous basons ici sur les résultats de PETIT et al. (1996).

$\mathrm{Au}$ Miocène supérieur et jusqu'au Pliocène inférieur (vers $-5 \mathrm{Ma}$; Figure 1), l'ensemble Aar + Rhin était un affluent du Danube, qu'il rejoignait au Nord du lac de Constance actuel. Suite aux mouvements tectoniques liés à la surrection des Alpes, le Rhin-Aar a suivi un nouveau cours, vers l'Ouest, débouchant dans le fossé rhénan, et s'écoulait ensuite vers le Nord (Figure 2), tandis que le Rhin « alpin » restait tributaire du Danube. Les mouvements de l'écorce terrestre se poursuivant, le Doubs a capturé l'Aar et une partie du Rhin (Rhin "supérieur »), qui longe alors le Jura vers le Sud et rejoint la Méditerranée (env. - $2 \mathrm{Ma}$ ). Le cours amont du Rhin (Rhin " alpin ») est resté un affluent du Danube, tandis que la plaine d'Alsace est drainée par un cours d'eau (Rhin « inférieur ») qui coule vers le Nord. 


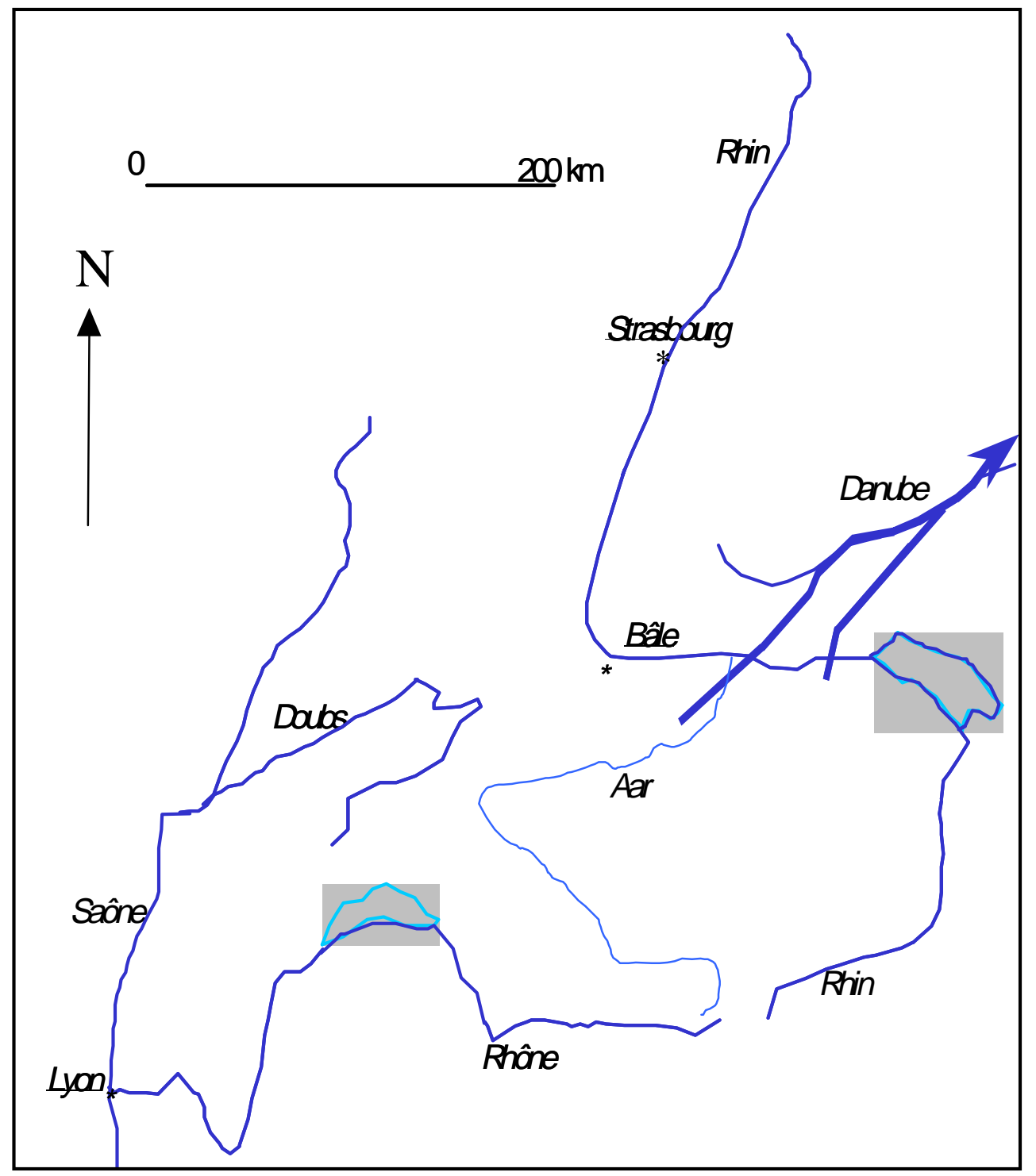

Figure 1

II y a plus de $5 \mathrm{Ma}$, l'ensemble Rhin + Aar était tributaire du Danube, où se trouvait S. glanis. L'espèce avait la possibilité de s'implanter dans ces affluents.

\section{Figure 1}

More than $5 \mathrm{My}$ ago, the Rhine with the Aar were flowing eastward and were tributaries of the Danube, from which S. glanis had the opportunity to colonize these rivers. 


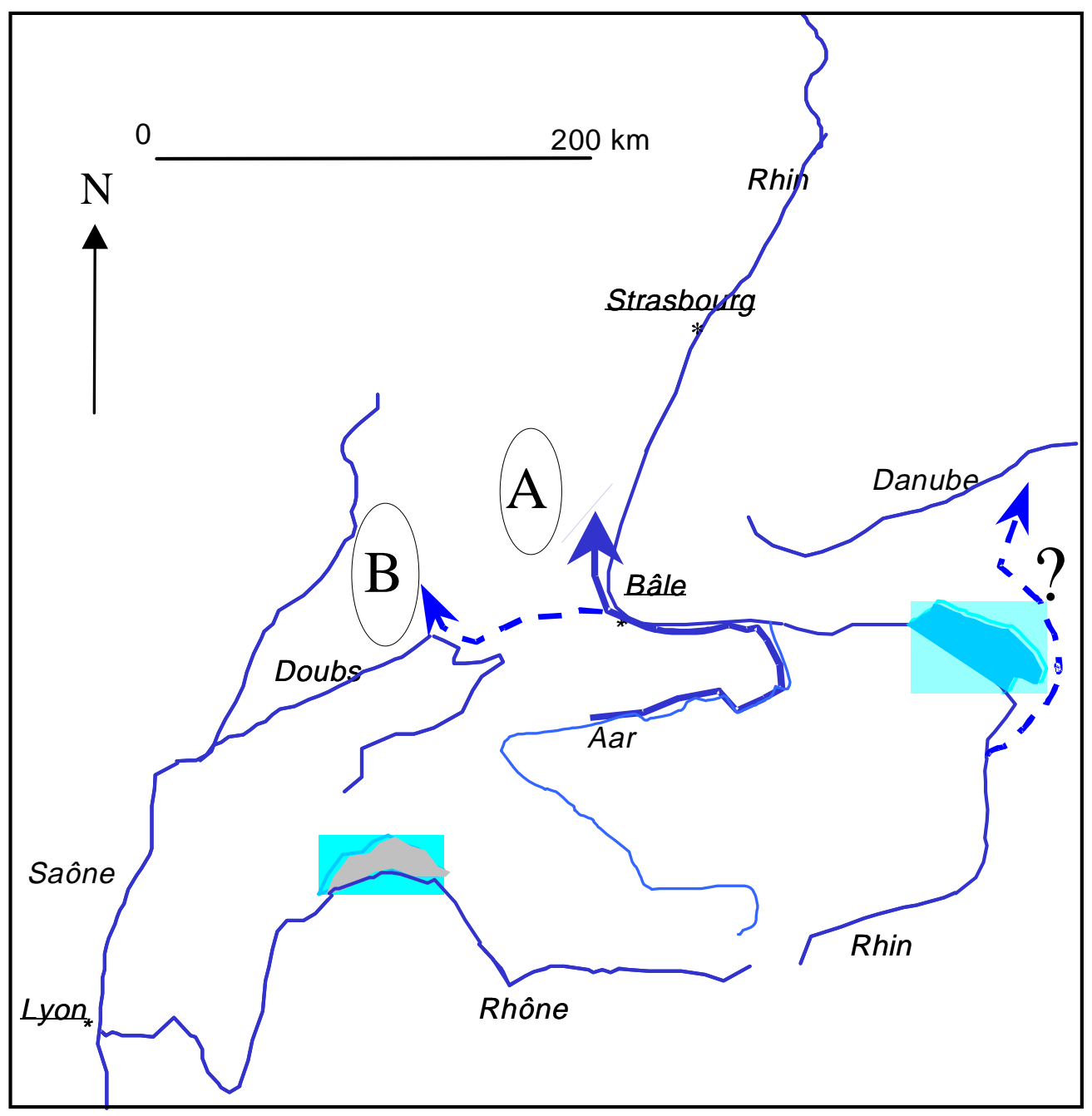

Figure 2

Des mouvements tectoniques ont affecté le fossé rhénan.

(A) : De -5 à $-3,2 \mathrm{Ma}$, Aar et Rhin " supérieur " coulent vers le Nord à travers la plaine d'Alsace. Ceci offre la possibilité au silure de coloniser la basse vallée du Rhin. Le Rhin « amont » reste affluent du Danube.

(B) : De -3,2 à $-2,6 \mathrm{Ma}$, le Rhin «moyen » avec l'Aar ont été capturés par le Doubs et deviennent des affluents du Rhône. Cette situation rend possible la colonisation de la vallée du Rhône par S. glanis. La plaine d'Alsace est drainée par le Rhin « inférieur ».

Figure 2

Tectonical mouvements affected the rift valley of the Rhine.

Between -5 to $-3.2 \mathrm{My}$, the Aar and the Rhine are flowing through the Alsace plain. S. glanis. had thus the opportunity to settle into the lower valley of the Rhine. The upper part of the Rhine remains a tributary of the Danube.

From -3.2 to $2.6 \mathrm{My}$, the medium course of the Rhine with the Aar where captured by the Doubs, becoming temporary tributaries of the Rhône. This pattern made possible the colonization of a new hydrographical basin by S. glanis. A « lower" Rhine was draining the Alsace plain. 
Puis vers la fin du Tertaire (-2 à $-1,5 \mathrm{Ma})$, le Sud de l'Alsace est le siège de mouvements tectoniques : exhaussement du Sundgau et surtout reprise de l'affaissement du fossé rhénan. En conséquence, Aar et «Rhin supérieur » sont capturés par le Rhin " inférieur » et s'écoulent vers le Nord ; on trouve une situation hydrographique similaire à la situation actuelle (Figure 3).

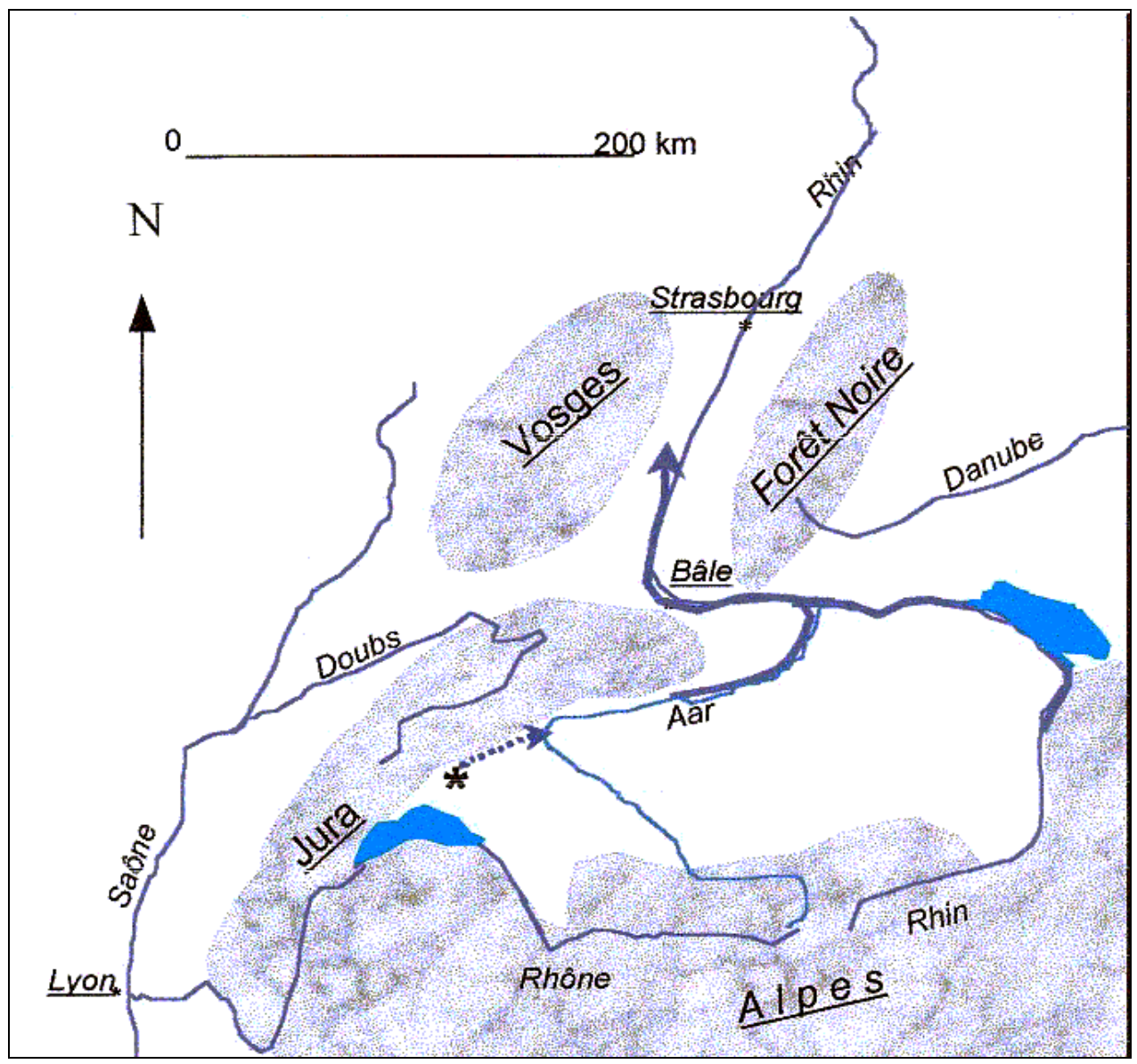

Figure 3

Vers la fin de l'ère Tertiaire (- 2,6 Ma), avant les grandes glaciations, un rehaussement de la bordure Nord du Jura provoque la capture de l'ensemble Rhin « moyen » + Aar par le Rhin « inférieur ». Cette situation a subsisté jusqu’à maintenant.

* : situation des lacs de Neuchâtel, Morat et Bienne, de formation post-glaciaire, où se trouvent de petites populations de $S$. glanis. Ces lacs font partie du réseau hydrographique de l'Aar.

\section{Figure 3}

Near the end of the Tertiary era (-2.6 My) and before the glaciations, a rise of the northern border of the Jura induced the capture of both the "upper " Rhine and the Aar by the « lower " Rhine. This situation has remained till nowadays.

* : Location of the lakes of Neuchâtel, Morat and Bienne, from post-glaciation origin, where small populations of $S$. glanis are existing. These lakes are connected to the Aar river.

L'écoulement temporaire de l'Aar (avec une partie du Rhin) vers le Doubs et le Rhône, a permis à la population rhénane de silure de coloniser ce nouveau réseau, en 
direction de la Méditerranée, comme l'attestent les restes trouvés en Ardèche. L'hypothèse d'un écoulement direct de l'Aar vers le Rhône au niveau du lac Léman actuel (TILLMANNS, 1984) n'est plus retenue, faute d'arguments géologiques.

Avant les grandes glaciations du Pléistocène, c'est à dire il y a environ - 1,8 Ma, des populations indépendantes de silure existaient :

- dans le bassin du Danube,

- dans celui du Rhône, matérielles.

- et probablement dans celui du Rhin, bien qu'on en n'ait pas retrouvé de preuves

Les quatre glaciations successives du Pléistocène qui ont recouvert de glaces l'Europe du Nord jusqu'aux Pays-Bas (jusqu'à 15000 ans avant JC), ainsi que tous les massifs montagneux, ont eu des effets radicaux sur la majeure partie de la faune qui ne pouvait rejoindre des régions plus méridionales.

Le glacier du Rhône descendit (temporairement) jusqu'à Lyon. Ces conditions climatiques extrêmes ont entraîné l'élimination du silure de l'ichtyofaune rhodanienne.

Sur le Rhin, son cours inférieur (à partir de l'Alsace actuelle) se dirigeant vers le Nord ne permettait pas la survie de l'espèce, avec un climat probablement similaire à celui du Nord de la Sibérie actuelle : à l'époque, la calotte glaciaire n'était qu'à guère plus de $500 \mathrm{~km}$. La persistance dans le delta du Rhin (selon BOESEMAN, 1975) d'une population ayant re-colonisé le bassin rhénan après y avoir survécu aux glaciations est une hypothèse difficile à accepter.

Les lacs suisses où subsistent actuellement de petites populations de silure, ainsi que le lac de Constance (où l'espèce est régulièrement pêchée), sont des plans d'eau de formation récente, post-glaciaire. L'extension de langues glaciaires d'origine alpine ayant interdit toute survie de l'espèce dans les bassins actuels du Rhin supérieur et de l'Aar, la présence du silure dans les lacs de Neuchâtel, Bienne et Morat ne peut être que d'origine anthropique (datant du Moyen Age ?). Elle ne démontre donc pas les anciennes relations entre Aar-Rhin et Danube, contrairement à ce qu'indique I' "Atlas de distribution des Poissons et Cyclostomes de Suisse » (PEDROLI et al., 1991).

L'absence d'espèces de poissons endémiques dans le bassin du Rhin (PEDROLI et al., 1991), et la subsistance d'une seule espèce endémique d'origine préglaciaire dans celui du Rhône (l'Apron du Rhône Zingel asper) indiquent bien l'effet radical des glaciations sur l'ichtyofaune initialement présente dans ces régions. Grâce aux variations des interconnexions entre bassins rhénan et rhodanien signalées précédemment, un ancêtre de Z. asper aurait colonisé le bassin du Rhône à partir du Danube (KEITH, 1998). Une hypothèse analogue peut être faite concernant la distribution du Blageon (Leuciscus (Telestes) soufia), présent seulement dans le bassin du Danube, dans celui du Rhône et dans les fleuves côtiers de Provence, qui ont pu constituer autant de refuges pendant les épisodes glaciaires.

Pendant ces périodes froides et jusqu'à la fin de la dernière glaciation (c'est à dire de - $2 \mathrm{Ma}$ à 12000 ans avant $\mathrm{JC}$ ), le maintien du silure n'était possible en Europe que sur le bassin du Danube, dans deux secteurs abrités des glaces (KEITH, 1998) :

- la plaine hongroise (Pannonie), située à la fois à l'écart des Alpes et des Carpathes, 
- la partie aval du fleuve, jusqu'à la mer Noire.

Des populations s'étaient également maintenues en mer Caspienne et en mer d'Aral, qui assurent par leurs tributaires, le lien avec la Russie et l'Asie.

\section{Comment le silure a-t-il atteint le Nord de l'Europe?}

(pourtours de la Baltique et delta du Rhin)

L'implantation post-glaciaire du silure au Nord de l'Europe est un exemple de colonisation rapide d'un milieu vierge où les conditions thermiques lui étaient plus favorables qu'actuellement. Les recherches en paléoclimatologie montrent qu'un réchauffement rapide et important a eu lieu vers 10000 avant JC (GIRARD, 1989), la Scandinavie bénéficiant de conditions thermiques estivales analogues à celles de la France actuelle (moyenne de juillet : $18^{\circ} \mathrm{C}$ ), mais avec des hivers nettement plus froids (LE MEUR, 1999).

Alors que le climat se réchauffe à partir d'il y a 12000 ans, très rapidement le silure est localisé :

- au Nord de l'Europe, dans le Sud de la Suède et de la Finlande ainsi qu'au Danemark à partir de la «période à Ancylus » (env. 6000 avant JC) et jusqu'au début du 19ème siècle (GILTAY, 1931).

- dans la Meuse et l'Escaut (datations entre 14000 et 5000 avant JC suivant les sites), dans le Rhin (à partir de 3500 avant JC), comme l'attestent les fouilles archéologiques (GILTAY, 1931; PHILIPPART, 1999). Il semble que la population ait peu à peu décliné à partir du XII ${ }^{\text {ème }}$ siècle, puis disparu, pour des raisons inexpliquées, probablement au XVIII ${ }^{\text {ème }}$ siècle (VOLZ, 1994). Bon naturaliste de son temps (milieu du $X V I I^{\text {eme }}$ S.), BALDNER ne connaissait pas le silure vu à Strasbourg ; il est donc permis de penser que déjà à l'époque, cette espèce était quasiment en voie de disparition dans le cours moyen du fleuve.

Précédé par une phase de refroidissement (XIII-XIV ${ }^{\text {èmes }}$ siècles), le "petit âge glaciaire » identifié par les historiens entre les années 1500 à 1850, a pu affaiblir des populations de silure mal implantées, relictuelles. Cette période plus froide coïncide en effet avec la disparition du Silure au Danemark et dans le Rhin moyen et aval. Le refroidissement de 1 à 2 degrés par rapport aux moyennes actuelles (MOBERG et DEMAREE, 1999) a probablement fragilisé la population vivant dans le Sud de la Finlande qui n'a pas survécu aux actions anthropiques sur son milieu.

Cantonné dans le Danube et l'Europe orientale après la fonte des glaces, le silure a pu bénéficier d'un réseau hydrographique encore confus qui lui aurait permis de passer soit du bassin hydrographique de la mer Noire (Dniepr), soit de celui de la Caspienne, (avec la Volga qui coule bien au Nord de Moscou) vers des cours d'eau ou des plans d'eau (lacs Onega et Ladoga) s'écoulant en direction du Nord de l'Europe, tributaires du « lac à Ancylus » (vers - 6000). Ce vaste lac occupait la mer Baltique actuelle ainsi que les golfes de Finlande et de Bothnie. II a permis au silure de coloniser ses rivages: Sud de la Scandinavie (où il subsiste localement), Danemark, ainsi que les fleuves côtiers des pays baltes, de Pologne et d'Allemagne du Nord. II est très probable qu'au cours du recul du front glaciaire, des interconnexions aient existé, à une époque ou une autre, entre le Rhin aval et des fleuves plus à l'Est (Oder, Vistule, tributaires de la mer Baltique) via l'Elbe.

Une autre possibilité serait la mise à profit d'interconnexions intra-continentales entre le bassin du Danube et celui de l'Elbe, dans le Sud de la Pologne et la Slovaquie. Les géographes indiquent en effet l'existence d'une longue dépression formée à l'avant du 
front glaciaire dont elle drainait les eaux de fonte, s'étendant suivant un arc Est-Ouest de Varsovie (sur la Vistule) à Hambourg (sur l'Elbe) via Berlin. Restée longtemps marécageuse, cette zone recoupe les bassins actuels de la Vistule, de l'Oder, et de l'Elbe, et a pu faciliter le passage du silure entre ces bassins hydrographiques. Ces deux hypothèses (passage par les rives $d u$ "lac à Ancylus » ou par des connexions entre réseaux hydrographiques à l'intérieur des terres) ne sont pas exclusives l'une de l'autre.

Bien que les preuves archéologiques fassent défaut, ce serait alors à partir de cette zone nord-européenne, que l'espèce aurait gagné vers l'Ouest le delta du Rhin, qui a constitué, pendant quelques millénaires (et avant de s'éteindre vers le XVIII ${ }^{\text {ème }}$ siècle), la limite occidentale de son aire d'extension naturelle.

Par contre, l'hypothèse d'une " dévalaison » post-glaciaire du silure le long du Rhin depuis la Suisse (comme le suggère VOLZ, 1994) est difficile à envisager dans l'état actuel des connaissances, faute de trouvailles archéologiques démontrant sa présence préhistorique dans l'Ouest de la Suisse et le Nord de l'Alsace (Rhin moyen) après une bien hypothétique survie aux glaciations.

Des conditions climatiques favorables et des réseaux hydrographiques instables ont facilité l'intrusion post-glaciaire du silure - et son implantation - dans la faune du Nord de l'Europe. La rapidité de son extension en quelques millénaires est assez remarquable, mais les observations montrent que dans un milieu neuf, le silure s'avère être un colon très actif. Par exemple, on note actuellement sur la Dordogne et la Garonne que de nombreux individus se déplacent vers l'amont dès que la température de l'eau dépasse $15^{\circ} \mathrm{C}$, et s'engagent avec succès dans divers dispositifs de franchissement de barrage (GALIAY, 1997).

A partir de ces données sur la biogéographie originelle du silure, il est possible de repérer les secteurs où l'action anthropique a joué un rôle, favorable ou non, à ce poisson.

\section{INFLUENCES ANTHROPIQUES}

Historiquement, sur la période 1850 - 1990, à la suite d'interventions anthropiques sur le milieu (dégradations) ou directement sur l'espèce (transfert d'individus), le silure voit sa distribution en Europe se décaler vers le Sud et le Sud-ouest.

Suite à des opérations $d^{\prime}$ " aménagement » de cours d'eau et de lacs, de destruction de végétation rivulaire (Salix, Carex, Phragmites), de pollution par les métaux lourds, l'espèce a disparu du Sud de Finlande vers 1865 et n'est plus présente que très ponctuellement dans quelques lacs et cours d'eau de Suède au Sud de Stockolm (NATHANSON, 1987 ; KOTIRANTA et al., 1998).

En France, après des essais sans suite dans les bassins de Versailles et du bois de Boulogne, des tentatives infructueuses ont eu lieu en Alsace à la fin du XVIIlème siècle par M.M. DURR, négociants en poissons à Strasbourg (LACEPEDE, 1803 ; BUFFON, 1804) et de DIETRICH, maire de Strasbourg (CUVIER et VALENCIENNES, 1839). Ces intervenants semblent avoir eu le même fournisseur allemand installé de l'autre côté de la Forêt Noire, à Donaueschingen (sur le cours supérieur du Danube). L'introduction du silure à partir du Danube n'a été effective qu'en 1857, dans la pisciculture de Huningue (Sud de l'Alsace). De là, il a atteint le bassin du Doubs vers 1890 (BROCCHI, 1896 ; GERVAIS, 1897) et rejoint le bassin hydrographique du Rhône par l'intermédiaire du canal du Rhône au Rhin ou grâce à des transferts directs.

L'intérêt à la fois halieutique et piscicole de l'espèce, qui s'est manifesté récemment, a facilité son extension en France à l'Ouest de l'axe Doubs-Rhône, dans les bassins de la 
Seine, de la Loire (deux captures en 1975 ; d'AUBENTON et SPILLMANN, 1975), puis de la Garonne après 1989 (ALLARDI et KEITH, 1991). La présence du silure dans la Saône résulte d'introductions dans des étangs piscicoles vers 1966 en Dombes (VALLOD, 1987). Quelques années plus tard, toujours dans un but d'élevage, l'espèce était introduite en Camargue, à la pisciculture de Sylvereal par son propriétaire d'origine hongroise (M. CORCHUS) dont la famille a joué un rôle de pionnier pour la pisciculture de ce poisson en Hongrie (UNGER, 1933). Dès les années 1976-78, des juvéniles étaient capturés au carrelet dans le petit Rhône, à Albaron.

Il a également été introduit en Grande-Bretagne vers 1880 par le duc de BEDFORD à l'Abbaye de Woburn, après une première tentative infructueuse vers 1862-65 à Reading (WEELER et MAITLAND, 1973), BROCCHI (1896) indiquant la pisciculture de Twickenham comme premier site d'entrée dans les îles britanniques.

Le silure glane est désormais présent au delà des Pyrénées : des amateurs de pêche sportive l'ont introduit vers 1990 dans une retenue sur l'Ebre : la Mequinenza, près de Barcelone. En Italie, on le trouve dans quelques secteurs de la plaine du Pô, apparemment depuis le début du XIX ${ }^{\text {ème }}$ siècle (BROCCHI, 1896). TORTONESE (1970) dans la «Fauna d'Italia » ne mentionne sa présence que comme exceptionnelle, avec la capture d'un individu femelle de 1,17 m vers 1936, mais les captures sont désormais de plus en plus fréquentes dans ce secteur (GANDOLFI et GIANNINI, 1979).

Depuis quelques années, il fait l'objet de réintroductions dans certains lacs du bassin de l'Elbe (Allemagne) dans un but de biomanipulation du milieu (réduction de la population de brèmes, Abramis brama) et de soutien à la pêche professionnelle (PARZYK et FLEMMIG, 1996 ; ANWAND et al., 1998).

Hors d'Europe, il faut signaler l'introduction récente du silure en Tunisie, dans les retenues de Sidi Salem, Sidi Saad, et Mellegue, suite à un programme germano-tunisien pour développer la pêche artisanale (LOSSE et al., 1992). Le silure a également été introduit en Algérie, pour les mêmes raisons. L'état de ces deux populations n'est pas connu.

En l'absence d'études qui démontreraient une concurrence interspécifique, il est probable que les capacités physiologiques du silure lui permettent de s'implanter dans un cours d'eau sans entrer en compétition avec les autres carnivores. Le brochet (Esox lucius) préfère les bordures de végétaux en eaux peu turbides et son régime alimentaire est plus strictement ichtyophage. Si le sandre (Stizostedion lucioperca) accepte les eaux turbides et les milieux profonds, il est plus oxyphile que le silure ; en outre, une phase de froid préalable lui est nécessaire pour une bonne reproduction (SCHLUMBERGER et PROTEAU, 1995). Des observations ponctuelles et non quantifiées signalent une réduction de l'abondance relative des brèmes (Abramis brama) dans des milieux où se développe le silure.

L'extension de l'aire de répartition du silure, d'origine anthropique, s'est faite apparemment en deux phases :

- au Moyen Age, où les poissons, grâce à la pêche et la pisciculture, fournissent une part importante de la nourriture pour les nombreux jours maigres du calendrier ; la rusticité de l'espèce et sa forte valeur marchande ont pu favorisé sa dispersion simultanément à celle des Cyprinidés ;

- au début du XIX ${ }^{\text {ème }}$ siècle, avec le développement des moyens de transport, l'intérêt agronomique et zoologique pour les « acclimatations » d'espèces diverses, et plus récemment, le goût de la "pêche au gros" en eau douce. Bien que ces transferts ne soient que rarement documentés en détail, ils n'ont pas toujours abouti à l'implantation du 
poisson dans son nouveau milieu. Ils révèlent incidemment l'intense activité des négociants en poissons, n'hésitant pas à transporter souvent sur de longues distances, des animaux vivants pour les introduire à proximité des marchés urbains.

Cette dispersion de l'espèce qui a pour foyer le bassin du Danube, s'est faite vers celui du Rhin : Sud de l'Allemagne (lac de Constance), lacs de Suisse (à l'exception du lac Léman), Alsace. On peut remarquer que cette extension à finalité économique, recouvre des régions où le silure a été (et est encore) considéré comme un poisson de choix. Actuellement, le silure vivant est le plus coté des poissons d'eau douce sur les marchés de Munich et Nuremberg : 30 - $40 \mathrm{DM} / \mathrm{kg}$ au détail, c'est à dire 3 à 4 fois plus cher que la carpe.

\section{STATUT DE L'ESPÈCE}

II faut rappeler qu'en Europe, mis à part les pays du bassin danubien, ainsi que la Pologne et la France, où l'espèce fait l'objet d'une production piscicole (environ 500 tonnes/an au total ; FAO, 1999), le silure est globalement une espèce menacée. Elle est classée en Annexe III de la Convention de Berne (Conseil de l'Europe, 1995 ; FIERS et al., 1997), au même titre, par exemple, que le saumon atlantique Salmo salar. Dans son aire de distribution, ne subsistent en effet que quatre populations isolées, relictuelles :

- en Suède, au Sud de Stockolm : le bassin du lac Baven, les cours inférieurs de l'Helgean et de l'Eman, avec quelques centaines d'individus en 1985 (NATHANSON, 1987), 1998),

- en Carelie russe : les lacs Ladoga, Onega et leurs tributaires (KOTIRANTA et al.,

- un site en Estonie : le lac Peipus (KOTIRANTA et al., 1998).

Le stock naturel présent dans le delta du Rhin (aux Pays-Bas, où l'espèce est strictement protégée) est peut être plus ou moins perturbé génétiquement (introgression) par des individus allochtones introduits ou échappés d'élevages (PHILIPPARD, 1999). Celui existant dans les lacs suisses du piedmont du Jura a une origine anthropique remontant vraisemblablement au Moyen Age, puisqu'on n'a pas connaissance d'introduction d'individus à une époque récente.

\section{CONCLUSION}

Les grandes tailles atteintes par le silure glane, sa présence dans les milieux lentiques et la solidité des pièces osseuses sont des facteurs favorisant la conservation et l'identification de restes archéologiques ou paléontologiques par rapport à d'autres espèces plus petites ou rhéophiles.

L'expansion post-glaciaire du silure à partir d'un foyer dans le Sud-Est de l'Europe (Danube, Dniepr, Volga) a été facilitée à la fois par une période d'optimum climatique et par ses caractères biologiques spécifiques (eurythermie, régime carnivore opportuniste avec détection des proies possible en eaux turbides, soins du mâle pour la ponte). Ces modalités de dispersion sont applicables dans une large mesure au sandre ( $S$. lucioperca), plus exigeant vis à vis des conditions de milieu, mais qui a les mêmes origines géographiques et dont les introductions en Europe occidentale, postérieures à celles du silure, ont obéi à des critères identiques (pisciculture, pêche). D'autres poissons ont probablement suivi les mêmes voies hydrographiques post-glaciaires pour (re-) coloniser 
l'Europe, mais des interventions anthropiques très anciennes (transport direct) masquent leur répartition originelle (cas de la perche ?, de certains Cyprinidés). On peut imaginer que le réchauffement climatique en cours puisse faciliter le maintien des populations les plus septentrionales en Europe, à condition qu'il n'y ait pas de dégradation (induite par le climat, ou d'origine anthropique directe) du milieu aquatique où elles subsistent.

Une telle étude biogéographique fait appel à des sources d'information variées qui doivent être recoupées entre elles pour s'enrichir mutuellement : paléontologie, archéologie, géologie, climatologie, physiologie et écologie de l'espèce... II est certain que des informations complémentaires seront apportées à l'avenir : des comparaisons génétiques restent à faire entre les différentes populations sauvages du Nord de l'Europe, de Suisse, du Danube, de la Volga. En outre, des documents restent encore à exploiter dans des archives diverses (professionnelles ou privées, commerciales ou scientifiques, de communautés civiles ou religieuses).

\section{REMERCIEMENTS}

Nous remercions tout particulièrement M. le Professeur H. VOGT, de la Faculté de Géographie de Strasbourg, de nous avoir transmis une documentation de base concernant la paléohydrographie du bassin supérieur du Rhin, puis d'avoir bien voulu relire le manuscrit pour y apporter des corrections et précisions. Nos remerciements vont également à J.C. PHILIPPART (Laboratoire de Démographie des Poissons et d'Aquaculture, Université de Liège, Belgique) qui nous a communiqué, avant publication, des rapports de recherches concernant l'historique de la présence du silure en Wallonie, ainsi que Messieurs HENNEQUART, pisciculteurs.

\section{BIBLIOGRAPHIE}

ALLARDI J., KEITH P. (coord.), 1991. Atlas préliminaire des poissons d'eau douce de France. Coll. Patrimoines naturels, vol. 4, MNHN-SFF/CSP/CEMAGREF/Min. Env., Muséum National d'Histoire Naturelle, Paris, 232 p.

ANWAND K., WYSUJACK K., VALENTIN M., 1998. Der Wels (Silurus glanis) : ein Wirtschaftsfisch des See- und Flussfischerei. Fischer und Teichwirt, $2:$ 47-51.

AUBENTON (d') F., SPILLMANN C. J., 1975. Le silure Silurus glanis Linné (1858). Piscic. Fr., 44 : 35-36.

BALDNER L., 1666. Vogel- Fisch- und Thierbuch (fac simile : Müller und Schindler Verlag, Stuttgart, RFA) in : Chefs d'oeuvre d'après nature ; trésors de la peinture naturaliste en Alsace. Catalogue de l'exposition, 158-159. Juin-octobre 1994, Strasbourg (France).

BOESEMAN M., 1975. De nederlandse Merval, Silurus glanis Linnaeus. Zool. Bijdr. 17 : 48-62.

BRANA J.Y., RIGAUD G., 1997. L'influence de l'Homme sur l'expansion du silure en Europe (Silurus glanis). Mémoire Univ. Lyon (France). 23 p. + annexes.

BROCCHI Dr. 1896 : La pisciculture dans les eaux douces. Bibliothèque des sciences et de l'industrie ; Librairies-imprimeries réunies, Paris. 320 p.

BUFFON G.L., 1804. Histoire générale et particulière des Poissons. (tome 12). F. Dufart impr., Paris.

CUVIER G., VALENCIENNES A., 1839. Histoire naturelle des Poissons (tome 14). PitoisLevrault, Paris.

Conseil de l'Europe, 1995 : Convention relative à la conservation de la vie sauvage et du milieu naturel en Europe. Série des traités européens, $n^{\circ} 104.34$ p.

F A O , 1999. Statistiques de la production de l'aquaculture. FIDI/C 815 (Rev. 11). FAO, Rome. 203 p. 
FATIO V., 1890. Faune des Vertébrés de la Suisse (vol. 5 : Poissons). Georg éd., Genève. $576 \mathrm{p}$.

FIERS V., GAUVRIT B., GAVAZZI E., HAFFNER P., MAURIN H., 1997 : Statut de la faune de France métropolitaine (statuts de protection, degrés de menace, statuts biologiques). Coll. patrimoines naturels, vol. 24. Services du Patrimoine Naturel / IEGB / Muséum National d'Histoire Naturelle de Paris / Réserves naturelles de France / Ministère de l'Environnement. Paris, $225 \mathrm{p}$.

FORGUE J., BURTIN B., MASSABUAU J.C., 1989 : Maintenance of oxygen consumption in resting Silurus glanis at different levels of ambiant oxygenation. J. exp. Biol., $143: 305-319$.

GALIAY E., 1997. Bilan des passages de silures aux stations de contrôle du bassin Garonne - Dordogne. Rapport Cemagref / Conseil Supérieur de la Pêche / INPENSEEIHT Toulouse. 5 p. + annexes.

GANDOLFI G., GIANNINI M., 1979. La presenza di Silurus glanis nel fiume Po (Osteichtyes Siluridae). Natura, $70: 3-6$.

GAUDANT P.J., 1994. L'ichtyofaune du Pannonien supérieur de Goetzendorf an der Leitha (Basse Autriche). Ann. Naturhist. Mus. Wien, 96 a : 117-131.

GERVAIS P., BOULART R., 1897. Les Poissons d'eau douce. J. Rotschild éd., Paris. $233 \mathrm{p}$.

GILTAY L., 1931. Note sur la présence en Belgique de Silurus glanis L. durant le Quaternaire. Bull. Mus. royal Hist. Nat. de Belgique, tome 7, 21: 1-7.

GIRARD M., 1989. La Terre se réchauffe, in : De Lascaux au Grand Louvre ; archéologie et histoire en France.(44-46). GOUDINEAU C. et GUILAINE J. coord. Errance éd., Paris. 265 p.

HARTMANN G. L., 1827. Helvetische Ichtyologie. Drell, Fûssli und Compagnie éd., Zurich.

HILGE V., 1985. The influence of temperature on the growth of the European catfish (Silurus glanis L.). J. Appl. Ichctyol., $1: 27-31$.

HORVATH L., TAMAS G., TÖLG I., 1984. Special methods in pondfish husbandry. Akademiai Kiado/Halver Corp. Seattle. HALVER J. E. éd., 146 p.

KEITH, P., 1998. Evolution des peuplements ichtyologiques de France et stratégie de conservation. Thèse, Univ. Rennes I. 236 p. + annexes.

KOTIRANTA H., UOTILA P., SULKAVA S., PELTONEN S.L., 1998. Red data book of east Fennoscania. Ministry of the Environment/Finnish Environment Institute/BotanicalFinnish Museum of Natural History, Helsinki, Finlande. 351 p.

LACEPEDE (de) E., 1803. Histoire naturelle des Poissons (tome 5). Plassan impr., Paris.

LE MEUR H., 1999. Comment l'homme a-t-il vécu la fin des glaciations ? La Recherche, $321: 62-63$.

LOSSE G.F., NAU M., WINTER M., 1992. Le développement de la pêche en eau douce dans le nord de la Tunisie. GTZ / CGP, Commissariat général à la pêche, Ministère de l'Agriculture, Tunis. $418 \mathrm{p}$.

MASSABUAU J.C., FORGUE J., 1995. Les capacités d'adaptation du silure glane en hypoxie : un cas exemplaire d'homéostasie du milieu intérieur. Aquat. Living Resour., $8: 423-430$.

MEIN P., MEON H., ROMAGGI J.P., SAMUEL E., 1983. La vie en Ardèche au Miocène supérieur d'après les documents trouvés dans la carrière de la montagne d'Andance. Nouv. Arch. Mus. Hist. nat. Lyon, fasc. 1 : 37-44.

MOBERG A., DEMAREE G., 1999. Ce que nous apprennent les thermomètres. La Recherche, $321: 69-71$.

MOHR E., 1957. Der Wels. Die neue Bücherei, A. Ziemsen Verlag, Wittenberg Lutherstadt. $44 \mathrm{p}$.

NATHANSON J. E., 1986. Malen - giganten i var sötvattenfauna. Fauna och flora, 81 : 179-180.

NATHANSON J. E., 1987. Malens utbredning i Sverige. Information from Institute of Freshwater Research, Swedish National Board, Drottingholm (Suède). № 1, $30 \mathrm{p}$.

NATHANSON J.E., 1989. Projecktet Malen. (Slutrapport 1982-86). Institute of Freshwater Research, Swedish National Board, Drottingholm (Suède). 33 p. 
NATHANSON J.E., GUSTAFSON R., OHLSON L., 1987. Malens Biotopval i Sverige (« habitat choice in the Sheatfish in Sweden »). Information from Institute of Freshwater Research, Swedish National Board, Drottingholm (Suède). № 8, 49 p.

PARZYK R., FLEMMIG J., 1996 : Die Bestandsentwicklung des europäischen Welses Silurus glanis Linné in der unteren Mittelelbe. Fischer undTeichwirt, $6: 235-238$.

PEDROLI J.C., ZAUGG B., KIRCHOFER A., 1991. Atlas de distribution des Poissons et Cyclostomes de Suisse. Documenta Faunistica Helvetica 11. Centre suisse de cartographie de la faune, Neuchâtel (Suisse). 206 p.

PERSAT H., KEITH P., 1997. La répartition géographique des poissons d'eau douce en France : qui est autochtone et qui ne l'est pas ?. Bull. Fr. Pêche Piscic., 344/345: 15-32.

PETIT C., CAMPY M., CHALINE J., BONVALOT J., 1996. Major palaeohydrographic changes in Alpine foreland during the Pliocene-Pleistocene. Boreas, 25 : 131-143.

PHILIPPART J.C., 1999. Les espèces de poissons non-indigènes de Wallonie. Situation en 1999 et perspectives. Communication au colloque GIPA «Quel avenir pour nos poissons en Wallonie ? ", La Marlagne (Wépion) 5-6 novembre 1999. Laboratoire de Démographie des Poissons et d'Aquaculture (LDPA) Univ. de Liège (Belgique).

PROTEAU J.P., SCHLUMBERGER O., ALBIGES C., 1994. Influence de la température sur la maturation gonadique finale du silure glane (Silurus glanis L.) : application a la reproduction hors saison naturelle. In : BASIL '94, Atelier international sur les bases biologiques de l'aquaculture des Siluriformes (résumés). Montpellier, 24-27 mai 1994. TEC et DOC Lavoisier, Paris. $194 \mathrm{p}$.

SCHLUMBERGER O., PROTEAU J. P., 1995. Reproduction of pike-perch (Stizostedion lucioperca) in captivity. J. Appl. Ichtyol. $12: 149-152$.

SCHLUMBERGER O., 1997. Mémento de pisciculture d'étang (3ème edition). Cemagref éditions. $237 \mathrm{p}$.

SCHMID J.,1996. Einiges über den Waller. Fischer und Teichwirt, $11: 463$.

TILLMANNS W., 1984. Dis Flussgeschichte der oberen Donau. Jh. geol. Landesamt Baden-Württenberg, 26 : 99-202.

TORTONESE E., 1970 : Fauna d'Italia, Osteichtyes ; vol. 1, 297. Ed. Calderini, Bologne. $565 \mathrm{p}$.

UNGER E., 1933. Le grand silure et son élevage par les carpiculteurs hongrois. Bull. Fr. Pisc., (V) 273-281.

VALLOD D., 1987. Le silure (Silurus glanis). ADAPRA (Lyon) / Association pour le Développement de l'Aquaculture (St André de Cubzac). Bulletin de l'ADA N 16. 70 p. + annexes.

VALLOT J. N., 1837. Histoire naturelle des Poissons d'eau douce de la France. Frantin impr., Dijon. $321 \mathrm{p}$.

VOLZ J., 1994. Über das naturliche Vorkommen des Welses (Silurus glanis) in Niederlandischen Rheindelta. Fischökologie, $7: 61-70$.

WEELER A., MAITLAND P.S., 1973. The scarcer freshwater fishes of the British Isles. 1. Introduced species. J. Fish Biol., 5 : 49-68. 